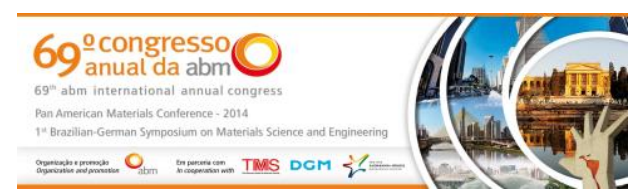

Tema: Materiais cerâmicos, compósitos e poliméricos

\title{
CARACTERIZAÇÃO DE CORREIAS TRANSPORTADORAS DE ALTA TEMPERATURA E AVALIAÇÃO DE SUA VIDA ÚTIL COM BASE NA DEGRADAÇÃO TÉRMICA DURANTE USO*
}

\begin{abstract}
André de Almeida Grilo ${ }^{1}$ Kinglston Soares ${ }^{2}$

Fábio Arielo Guastala ${ }^{3}$

\section{Resumo}

Correias transportadoras de alta temperatura são ativos críticos para a pelotização devido ao prejuízo que sua falha pode trazer para a produção. Nesse sentido, deve-se avaliar a vida útil da correia em uso, visando, principalmente, auxiliar sua manutenção, e nesse contexto o presente trabalho tem como proposta utilizar a caracterização dos componentes e o estudo da degradação térmica do material da cobertura de correias transportadoras de alta temperatura. As caracterizações foram realizadas de modo comparativo entre três tipos distintos de correias transportadoras de alta temperatura fornecidas para a VALE S.A. Os reforços das correias foram caracterizados por FT-IR para análise qualitativa do polímero. De maneira semelhante, as borrachas foram caracterizadas por meio da análise do produto de pirólise via FT-IR e a densidade de ligações cruzadas por meio da técnica de inchamento do polímero e aplicação da equação de Flory-Rhener, o que ajudou a justificar o comportamento das diferentes marcas de correia perante a degradação térmica, estudada em um trabalho anterior. A partir desses resultados e utilizando dados de variação das propriedades mecânicas do material em função do tempo e temperatura de processo, pôde-se avaliar a taxa de dano causado a cada correia e, aplicando-se dados reais de processo, avaliou-se 0 desempenho das correias em trabalho.
\end{abstract}

Palavras-chave: Correias transportadoras; EPDM; FT-IR; Equação de Flory-Rehner.

\section{CHARACTERIZATION OF HEAT RESISTANT CONVEYOR BELTS AND EVALUATION OF THEIR LIFE CYCLE BASED ON THERMAL DEGRADATION DURING WORK \\ Abstract}

Heat resistant conveyor belts are critical assets for a pelletizing plant because of the losses that their failures cause. Accordingly, the life cycle of these conveyor belts should be evaluated during work, in order to assist their maintenance, and, in this context, this study proposes the use of material characterization and thermal degradation of the conveyor belts components. The characterizations were performed in a comparative way between three different kinds of heat resistant conveyor belts supplied to a global mining company. The belts carcasses were characterized by FT-IR aiming at the polymers qualitative analysis. Similarly, the rubbers were characterized by the analysis of their pyrolyzed products by FT-IR and their crosslinks densities were evaluated by the use of the Flory-Rehner equation after the swelling of the rubbers, which helped to justify the belts behavior under thermal degradation, studied in a previous work. Using these results and data of mechanical properties variation according to time and process temperature, it was possible to establish the rate of damage done to each conveyor belt and, applying real process data, the belts performance during work could be evaluated.

Keywords: Conveyor belts; EPDM; FT-IR; Flory-Rehner equation.

1 Engenheiro Metalurgista, Instituto Federal do Espírito Santo (IFES), Vitória, ES, Brasil.

2 Doutor em Ciência e Engenharia de Materiais, Professor, Coordenadoria de Engenharia Metalúrgica, Instituto Federal do Espírito Santo (IFES), Vitória, ES, Brasil.

3 Mestre em Engenharia de Materiais, Engenheiro de Materiais, Gerência de Manutenção Mecânica da Pelotização, Vale S.A., Vitória, ES, Brasil.

\footnotetext{
* Contribuição técnica ao $69^{\circ}$ Congresso Anual da ABM - Internacional e ao 14ํㅡㄹ ENEMET - Encontro Nacional de Estudantes de Engenharia Metalúrgica, de Materiais e de Minas, 21 a 25 de julho de 2014, São Paulo, SP, Brasil.
} 


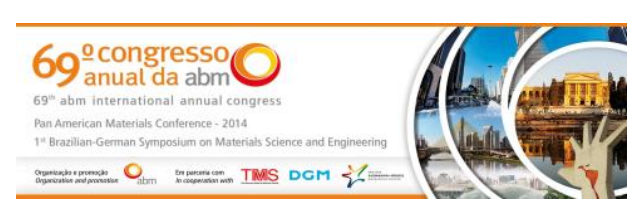

\section{INTRODUÇÃO}

Correias transportadoras são arranjos de componentes destinados à movimentação ou transporte de materiais a granel [1]. Por se tratar de um transporte de material de fluxo contínuo e ser um dos ativos mais custosos para a operação, o acompanhamento da vida útil da correia aliado ao conhecimento dos mecanismos de desgaste que atuam no seu material torna-se importante para minimizar prejuízos e perdas de produção [2, 3]. O presente caso leva em conta correias transportadoras de alta temperatura da saída do forno de pelotização e, como em uma usina de pelotização não se é utilizada uma correia de um único fornecedor, devem-se identificar as diferenças entre os materiais utilizados pelos diferentes fabricantes, o que certamente influenciará na degradação térmica e, portanto, a vida útil da correia. Inicialmente, deve-se levar em conta que as correias são constituídas por duas partes, a carcaça e a cobertura [1, 4], e, neste caso, a cobertura é de borracha e necessita apresentar ótimas propriedades térmicas e mecânicas, o que leva à utilização do EPDM [5-13]. Para as carcaças, geralmente são utilizadas fibras sintéticas como poliéster, nylon ou a composição dos dois, visando obter alta resistência à tração e fadiga [4, 13-15].

Para atestar a qualidade das correias fornecidas, seus componentes foram caracterizados. Identificaram-se os materiais poliméricos, tanto das fibras como das borrachas e, adicionalmente, promoveu-se $o$ inchamento das borrachas para 0 cálculo de suas densidades de ligações cruzadas. Com esses resultados, foi justificado o comportamento desses mesmos tipos de correia sob desgaste termomecânico, avaliado em estudo anterior [11]. Com os dados de desgaste termomecânico [11] e dados reais de processo, foi elaborado um modelo comparativo entre o desempenho das correias durante seu uso, a partir da avaliação de sua vida útil.

\section{MATERIAIS E MÉTODOS}

\subsection{Materiais}

Os materiais estudados no presente trabalho foram três amostras de correia transportadora de alta temperatura utilizadas nas saídas de fornos de pelotização, todas fornecidas pela mineradora Vale S.A. As correias foram denominadas A, B e $C$, sendo que cada uma possui uma marca registrada distinta e que as correias $\mathrm{B}$ e C são de um mesmo fornecedor. De cada correia foram extraídas amostras da cobertura superior e das fibras das carcaças presentes na direção do movimento das correias (Figura 1).

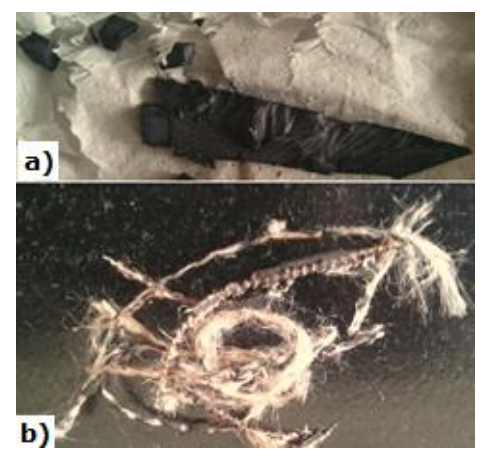

Figura 1. Amostras de a) cobertura superior e b) fibras de carcaça.

\footnotetext{
* Contribuição técnica ao $69^{\circ}$ Congresso Anual da ABM - Internacional e ao 14ํㅡㄹ ENEMET - Encontro Nacional de Estudantes de Engenharia Metalúrgica, de Materiais e de Minas, 21 a 25 de julho de 2014, São Paulo, SP, Brasil.
} 


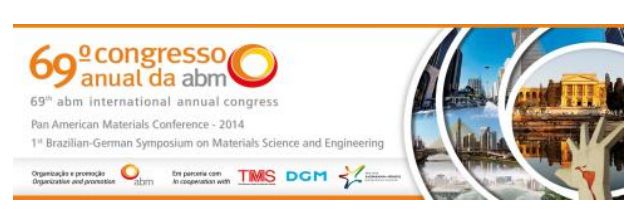

transferidas para tubos de ensaio. Então, adicionou-se aproximadamente $10 \mathrm{~mL}$ de n-heptano e cobriu-se cada tubo com papel alumínio para evitar evaporação. Deixou-se o sistema em descanso por um dia para que o inchamento atingisse 0 equilíbrio. Para cada amostra, após o equilíbrio, retirou-se a borracha do solvente e enxugou-se a superfície com papel absorvente. Rapidamente, colocou-se a amostra em uma balança e acompanhou-se a redução de seu peso com o tempo, devido à evaporação do solvente. Com aproximação de quatro casas decimais, tomou-se nota do peso da borracha inchada a cada dez segundos de pesagem, por 160 segundos. Plotou-se, então, um gráfico peso x tempo, extrapolando-se as curvas para $t=0$, quando $o$ valor do peso era o real da amostra inchada. Com essa informação, pode-se calcular $\mathrm{V}_{\mathrm{B}}$.

O n-heptano foi escolhido pois é um solvente do EPDM. Sabe-se que sua massa molecular é de 100,21 $\mathrm{g} / \mathrm{mol}$ e que sua densidade vale $0,684 \mathrm{~g} / \mathrm{cm}^{3}$. Com essas duas informações, pode-se calcular tanto $V_{B}$, como $V_{0}$. Além disso, segundo a literatura, o parâmetro de Flory para o sistema EPDM/n-heptano varia em torno de 0,4 [19, 28-31].

Desse modo, a única variável restante a ser determinada é $\rho_{\mathrm{B}}$, a densidade da borracha. Como as borrachas em geral, incluindo o EPDM, possuem várias grades, podem conter diversos aditivos e apresentar diferentes graus de cristalinidade, então também pode apresentar densidades distintas, apesar de ser o mesmo material. Nesse caso, a determinação da densidade necessita de uma técnica mais sensível de determinação de massa e volume, portanto outro experimento foi realizado.

\subsubsection{Densidade da borracha}

A determinação da densidade da borracha foi realizada por picnometria, uma técnica precisa e sensível. Esse experimento foi realizado para duas amostras de borracha de cada correia e, portanto, utilizaram-se dois picnômetros. Inicialmente, calibraramse os picnômetros para determinação da capacidade volumétrica dos mesmos [32]. A seguir, determinou-se a densidade das borrachas a partir da variação no volume de água do picnômetro sem a adição de amostras (calibração) e com a adição de amostras [32].

A diferença entre a massa do picnômetro vazio e do picnômetro com a amostra determina a massa da amostra. Já a diferença entre a massa do picnômetro com amostra e água destilada e do picnômetro com amostra determina a massa da água destilada. $O$ volume da água é então determinado pela relação entra a massa da água e a densidade da água, obtida na calibração do picnômetro. A capacidade volumétrica do picnômetro, informação obtida em sua calibração, subtraída do volume da água, determina o volume da amostra de borracha. Finalmente, a densidade da borracha pode ser calculada fazendo-se a razão da massa da amostra de borracha com o seu volume. A densidade considerada para cada borracha de cada correia foi a média das densidades obtidas para as duas amostras ensaiadas.

\subsection{Desempenho em Serviço}

Para realizar essa análise, foi recorrida à literatura de Guastala [11], que também é a base deste trabalho, pois ela contém resultados de ensaios de envelhecimento térmico para as mesmas correias aqui estudadas. Esses ensaios geraram como resultado curvas de \%Dano em função de tempo e temperatura para cada correia (Figura 2). Segundo Guastala [11], a \%Dano é a perda de resistência à tração da amostra ensaiada em relação à amostra virgem. Além disso, as correias $\mathrm{B}$ e $\mathrm{C}$, a

* Contribuição técnica ao 69 Congresso Anual da ABM - Internacional e ao 14ํㅡㄹ ENEMET - Encontro Nacional de Estudantes de Engenharia Metalúrgica, de Materiais e de Minas, 21 a 25 de julho de 2014, São Paulo, SP, Brasil. 


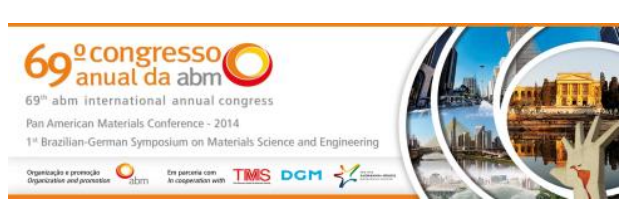

temperaturas mais baixas e tempos de exposição menores apresentam uma \%Dano negativa, ou seja, um ganho na resistência mecânica. Isso, provavelmente, se deve a uma sequência de vulcanização, com formação de novas ligações cruzadas antes da degradação do polímero.

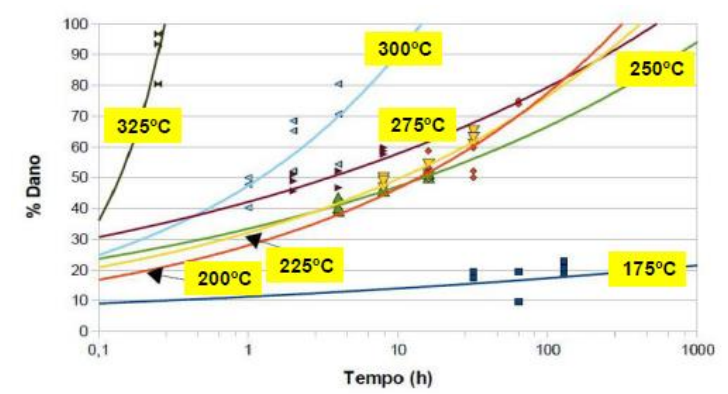

Figura 2. Exemplo de curva de dano em função de tempo e temperatura de envelhecimento acelerado para a correia $A$, uma das correias estudadas em [11].

Como o dano causado às correias pela degradação térmica é cumulativo, já que, na degradação polimérica, uma vez que se rompe uma ligação ela não se regenera, e levando em consideração que as temperaturas de trabalho não são mantidas em constância, como no caso dos ensaios de envelhecimento térmico, foi proposto que, para que as exponenciais obtidas experimentalmente por Guastala [11] fossem facilmente aplicadas a dados reais, elas deveriam ser aproximadas a retas, aplicando-se algumas modificações nas estruturas dos gráficos. Isso porque, dessa maneira, a taxa de acúmulo de dano, ou seja, o dano causado por certa temperatura somado ao dano causado por uma temperatura em um instante anterior, tanto em tempos de exposição menores ou maiores, seria a mesma. A partir das equações de reta encontradas, foram aplicados dados de temperatura de pelotas recém-saídas do forno de pelotização fornecidos pela Vale, e que também podem ser encontrados em Guastala [11], com um fator de 0,425 multiplicado a elas, para aproximar a temperatura das pelotas às temperaturas reais na superfície das correias, visando comparar, em um mesmo intervalo de tempo, como o desgaste térmico danifica os três tipos de correia. Essa comparação foi baseada nos resultados das caracterizações e pôde atestar se o ajuste das equações foi válido.

\section{RESULTADOS E DISCUSSÃO}

\subsection{Caracterização das Fibras da Correia A}

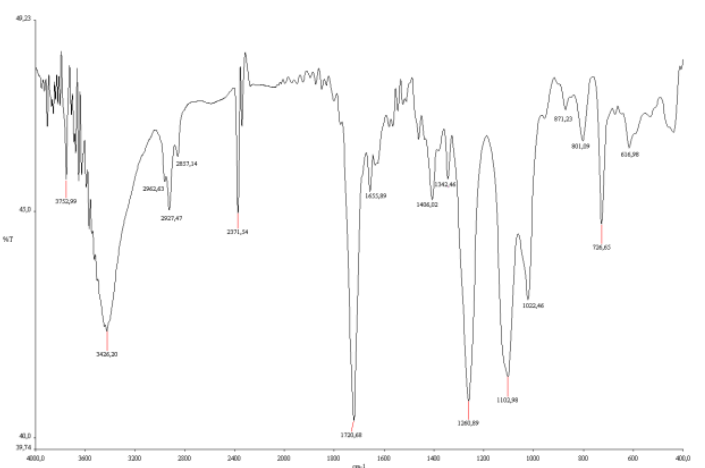

Figura 3. Espectro de FT-IR das fibras da correia A.

\footnotetext{
* Contribuição técnica ao $69^{\circ}$ Congresso Anual da ABM - Internacional e ao 14ํㅡㄹ ENEMET - Encontro Nacional de Estudantes de Engenharia Metalúrgica, de Materiais e de Minas, 21 a 25 de julho de 2014, São Paulo, SP, Brasil.
} 


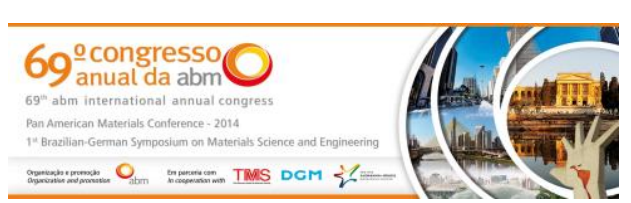

Analisando o espectro obtido (Figura 3), confirma-se que trata-se de um poliéster. Isso fica evidente devido, principalmente, à presença dos picos devido a excitações nas regiões de número de onda de valor 3400 cm-1 e 2900 cm-1 (ligação C-H), 1700 cm-1 (ligação $\mathrm{C}=\mathrm{O}$ ), $1650 \mathrm{~cm}-1$ (ligações $\mathrm{C}=\mathrm{C}$ no anel) e $1260 \mathrm{~cm}-1$ (ligações $\mathrm{O}-\mathrm{C}=\mathrm{O}$ ) [33, 34]

Comparando-se o espectro obtido pela análise da amostra da correia A com o espectro de um PET da literatura (Figura 4) [35], observam-se os mesmos picos característicos, o que atesta sua fabricação em PET. Fibras de PET na direção do movimento das correias proporcionam a estas uma alta resistência à tração aliada à boa resistência à abrasão e à fadiga, alta estabilidade dimensional e baixo alongamento, essenciais para essa aplicação.

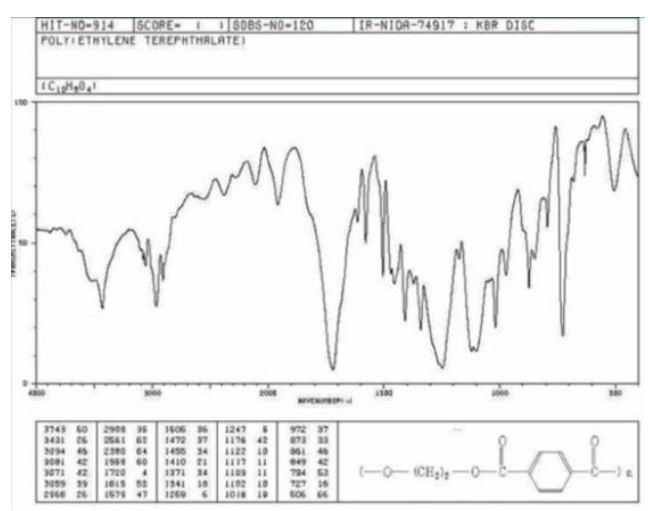

Figura 4. Espectro de FT-IR do PET [35].

\subsection{Caracterização das Fibras das Correias B e C}

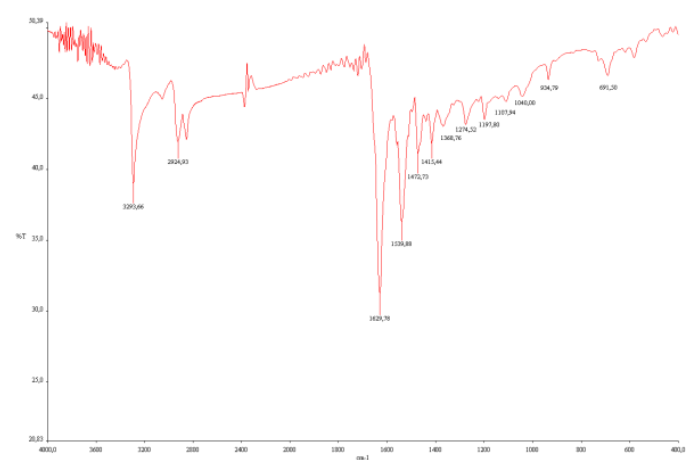

Figura 5. Espectro de FT-IR das fibras da correia B.

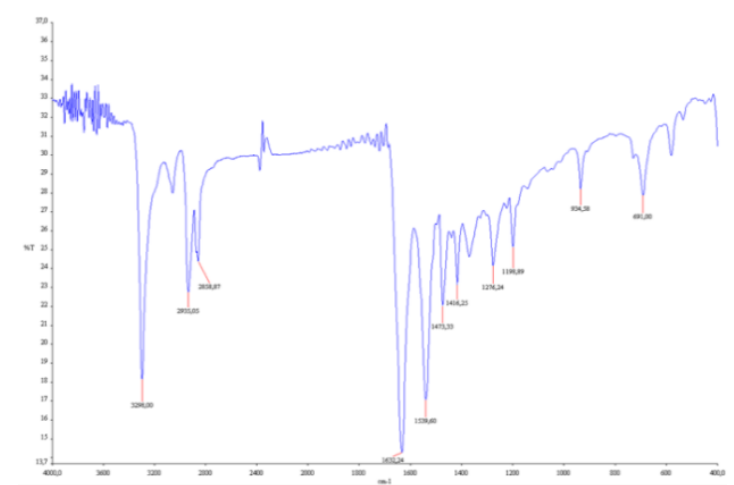

Figura 6. Espectro de FT-IR das fibras da correia C.

\footnotetext{
* Contribuição técnica ao $69^{\circ}$ Congresso Anual da ABM - Internacional e ao 14ํㅡㄹ ENEMET - Encontro Nacional de Estudantes de Engenharia Metalúrgica, de Materiais e de Minas, 21 a 25 de julho de 2014, São Paulo, SP, Brasil.
} 

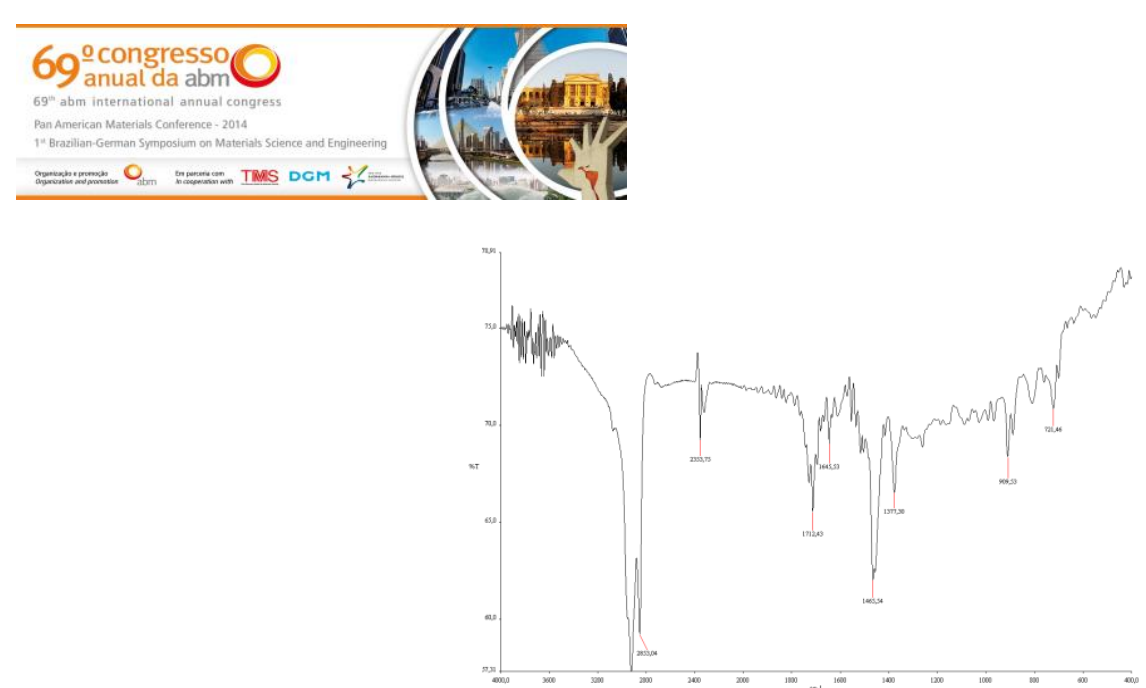

Figura 9. Espectro de FT-IR do pirolisado líquido da cobertura da correia B.

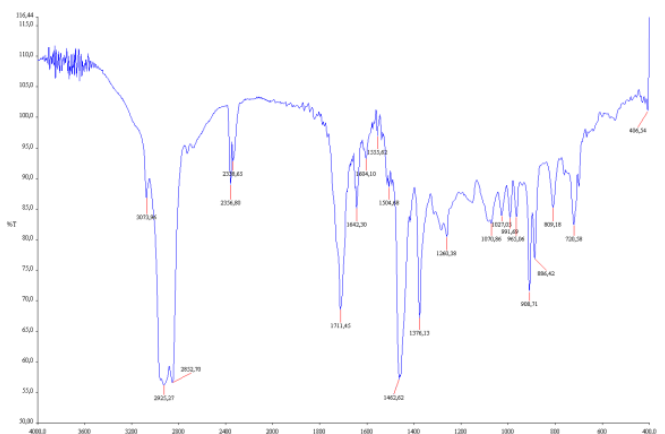

Figura 10. Espectro de FT-IR do pirolisado líquido da cobertura da correia C.

\subsection{Picnometria}

A partir dos cálculos de picnometria realizados, as densidades das borrachas provenientes das coberturas das correias $\mathrm{A}, \mathrm{B}$ e $\mathrm{C}$ foram determinadas, em que a densidade da borracha da correia $A$ foi de $1,207 \mathrm{~g} / \mathrm{cm}^{3}$, enquanto que a densidade da borracha da correia B foi de $1,113 \mathrm{~g} / \mathrm{cm}^{3}$ e a da correia $C$ de $1,044 \mathrm{~g} / \mathrm{cm}^{3}$.

\subsection{Determinação da Densidade de Ligações Cruzadas}

Com os valores de densidade das borrachas, todas as variáveis puderam ser aplicadas na equação de Flory-Rehner (Equação 1), obtendo-se resultados médios de densidades de ligações cruzadas de $1,808 \times 10^{-4}$ para a correia $A$, de $4,563 \times 10^{-5}$ para a correia B e de $3,864 \times 10^{-5}$ para a correia C.

Esses valores bastante superiores da correia $A$ em relação às correias $B$ (aproximadamente 4 vezes maior) e C (aproximadamente 5 vezes maior) significam que a cobertura da correia $A$ é certamente mais dura, rígida e elástica quando comparada às outras duas. Além disso, esses valores também dão indícios de que a correia A possui uma resistência inicial ao aporte térmico superior.

Levando em consideração esses resultados, analisando os espectros obtidos para os pirolisados líquidos do EPDM - em que os espectros de B e $C$ são mais parecidos do que o espectro $A$ - e sabendo que as correias $B$ e $C$ são produzidas por um mesmo fabricante, infere-se que o processo de fabricação das borrachas de $\mathrm{B}$ e $\mathrm{C}$ é muito semelhante e, por isso, os resultados obtidos para ambos são parecidos, quando comparados com os resultados e o processo de fabricação da borracha de A.

\footnotetext{
* Contribuição técnica ao $69^{\circ}$ Congresso Anual da ABM - Internacional e ao 14ํㅡㄹ ENEMET - Encontro Nacional de Estudantes de Engenharia Metalúrgica, de Materiais e de Minas, 21 a 25 de julho de 2014, São Paulo, SP, Brasil.
} 


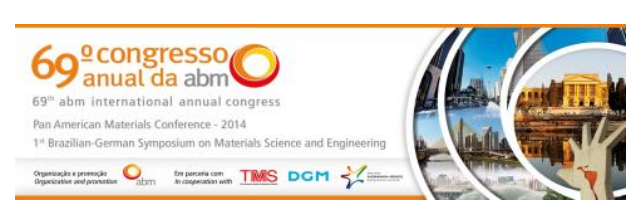

\subsection{Desempenho em Serviço}

Os gráficos de Guastala [11], originalmente plotados em função de \%Dano e tempo em horas, foram modificados para serem plotados em função de log \%Dano e log tempo em minutos. Além disso, foi traçada uma regressão linear que representou a taxa de acúmulo de dano nas correias (Figuras 11, 12 e 13).

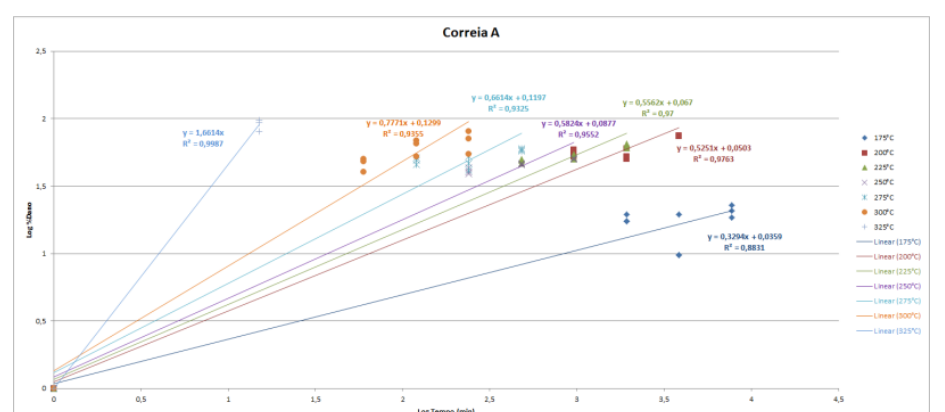

Figura 11. Gráfico do log \%Dano com regressão linear para a correia A.

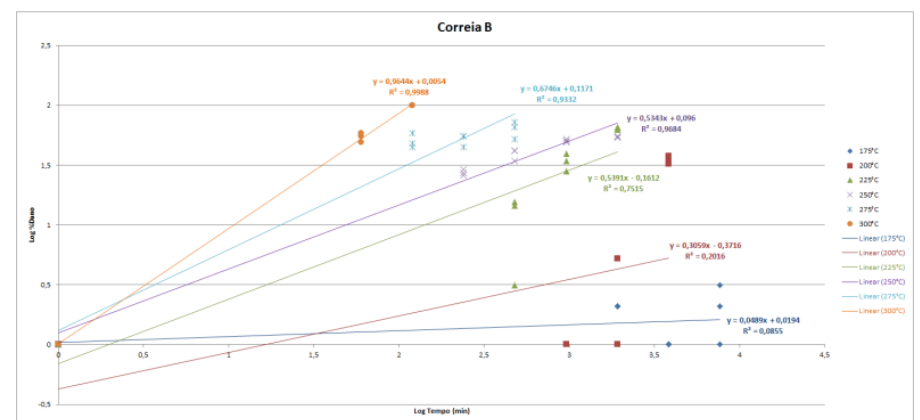

Figura 12. Gráfico do log \%Dano com regressão linear para a correia B.

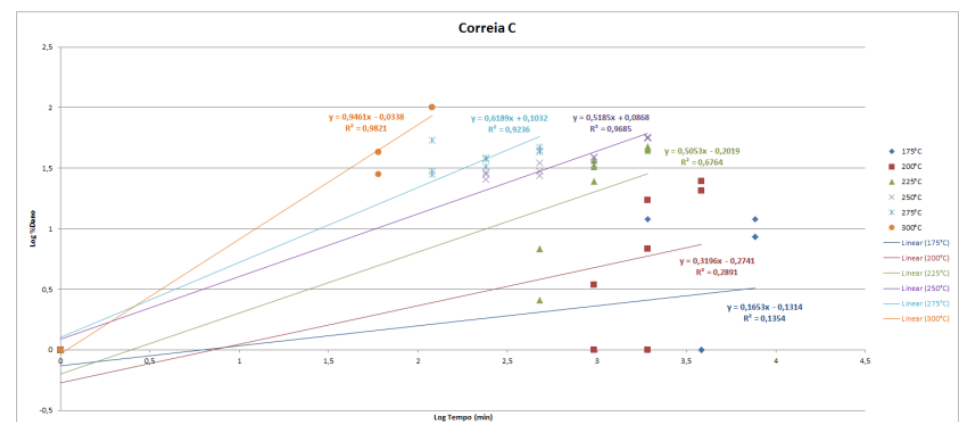

Figura 13. Gráfico do log \%Dano com regressão linear para a correia C.

Com a aplicação de 41536 dados de medição de temperatura das pelotas coletados entre 01/11/2010 e 15/11/2010 e aplicando-se um fator de 0,425 nessas temperaturas, para representar as temperaturas na superfície das correias, verificouse que em $79,6 \%$ do tempo de exposição, as equações que regem o dano nas correias são aquelas que dizem respeito a temperaturas da ordem de $175^{\circ} \mathrm{C}$. Os picos da ordem de 200 ocorreram em $0,3 \%$ do tempo. Em 20,1\% do tempo a temperatura encontrou-se abaixo dos $75^{\circ} \mathrm{C}$ e não foi considerada degradação. Dessa maneira, segundo as regressões lineares, após os 15 dias de medições, a correia A teria uma \%Dano de $255,5 \%$, a correia B, de $2,5 \%$ e a correia $C$ de $7,2 \%$.

Claramente, pelo resultado da correia $A$, o método de regressão linear utilizando logaritmos não foi satisfatório como modelo de cálculo da \%Dano das correias.

* Contribuição técnica ao 69 Congresso Anual da ABM - Internacional e ao 14ํㅡㄹ ENEMET - Encontro Nacional de Estudantes de Engenharia Metalúrgica, de Materiais e de Minas, 21 a 25 de julho de 2014, São Paulo, SP, Brasil. 


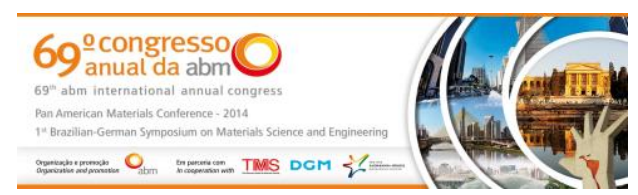

Entretanto, qualitativamente, este modelo também comprova a superioridade das correias $\mathrm{B}$ e $\mathrm{C}$ em relação à correia $\mathrm{A}$.

\section{CONCLUSÃo}

Para a correia $A$, identificou-se que suas fibras são produzidas em PET, enquanto que as fibras das correias B e C são produzidas em Nylon 6,6. Em termos de resistência ao aporte térmico, as fibras não são determinantes, mas são importantes no que diz respeito à resistência mecânica da correia, principalmente porque as fibras retiradas estavam dispostas no sentido de movimento da correia. Fibras de Nylon 6,6 alta resistência à tração, com boa resistência à fadiga e ao impacto. Entretanto, apresentam grande alongamento e baixa estabilidade dimensional. Já as fibras de PET também apresentam alta resistência à tração e fadiga, porém possuem baixo alongamento e grande estabilidade dimensional. Essas características fazem com que as fibras de PET sejam mais indicadas para a direção de movimento da correia, onde as forças trativas são fortes atuadoras, o que exige que a correia mantenha sua estrutura firme para suportar as condições de trabalho.

No caso da análise por FT-IR das borrachas, todas as amostras de cobertura das três correias estudadas foram identificadas como sendo fabricadas em EPDM, como esperado. A diferença entre as três se mostrou na determinação da densidade de ligações cruzadas das borrachas, em que a borracha da correia $A$ apresentou valor aproximadamente 4 vezes maior do que a da correia $B$ e aproximadamente 5 vezes maior do que a da correia $\mathrm{C}$. Esses resultados indicaram uma maior dureza, rigidez, elasticidade e resistência ao aporte térmico da borracha da correia $A$ em relação às outras duas. Entretanto, a temperaturas baixas e baixos tempos de exposição, as borrachas das correias B e C sofrem uma vulcanização adicional, gerando mais ligações cruzadas e, portanto, aumentando sua resistência mecânica. Em consulta à literatura em Guastala [11] e De Paoli [38] e na análise do desempenho em serviço, verificou-se que os valores inferiores de densidade de ligações cruzadas das borrachas das correias B e C são, ou devidos ao processo de fabricação, que não permite uma vulcanização completa; ou a um maior teor de etileno no EPDM, já que a degradação de cadeias semelhantes ao polietileno ocorre por abstração de hidrogênio, o que pode gerar ligações cruzadas antes da cisão de cadeiass. Dessa maneira, enquanto a degradação da correia $A$ começa no instante em que sofre influência da temperatura, as correias B e C ainda passam por um período de fortalecimento de sua estrutura, antes de sua degradação começar.

Apesar de ter sido importante qualitativamente na análise do comportamento de criação de novas ligações cruzadas durante o uso pelas correias $\mathrm{B}$ e $\mathrm{C}$, a análise do desempenho em serviço das correias pela regressão linear do logaritmo da \%Dano não foi satisfatória quantitativamente, visto o resultado irreal para a correia $A$. Isso ocorreu pois não foi levada em conta a questão cinética da degradação polimérica, em que a tempos baixos de exposição a taxa de degradação é muito baixa e, à medida que o tempo aumenta, esta taxa aumenta cada vez mais. A utilização de uma média de acúmulo e dano não conseguiu representar essa variação da taxa de dano fielmente. Além disso, a utilização de temperaturas das pelotas não representa fielmente as temperaturas às quais as superfícies das correias estão sujeitas durante o uso.

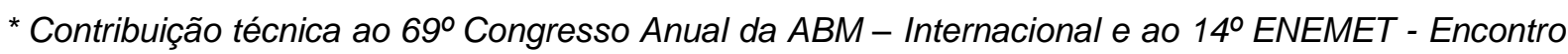
Nacional de Estudantes de Engenharia Metalúrgica, de Materiais e de Minas, 21 a 25 de julho de 2014, São Paulo, SP, Brasil.
} 


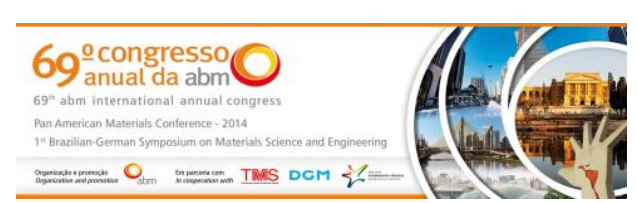

21 Flory PJ. Thermodynamics of high polymer solutions. The Journal of Chemical Physics. 1942; 10: 51-61.

22 Flory PJ; Rehner JJ. Statistical mechanics of cross-linked polymer networks. The Journal of Chemichal Physics, 1943; 11: 521-526.

23 Omidian H, Hashemi S, Askari F, Nafisi S. Swelling and crosslink density measurements for hydrogels. Iranian Journal of Polymer Science and Technology, 1994; 3(2): 115-119.

24 Peppas NA. Kinetics of smart hydrogels. Reflexive Polymers and Hydrogels: Understanding and Designing Fast-responsive Polymeric Systems, Yui N; Mrsny R; Park K. Boca Raton: CRC Press; 2004: 99-113.

25 Mahomed A. Properties of elastomers for small-joint replacements [Doutorado]. Birmingham: The University of Birmingham, 2008.

26 Cates RS. Influence of crosslink density on swelling and conformation of surfaceconstrained poly(n-isopropylacrylamide) hydrogels. Flórida: University of South Florida, 2010.

27 Huggns ML. Properties of rubber solutions and gels. Industrial and Engineering Chemistry. 1943; 35: 216-220.

28 Knibbe DE. Diffusion-controlled stress relaxation of swollen rubber-like networks [Doutorado]. Roterdam: Universitaire pers Rotterdam, 1968.

29 Hrnjak-Murgic Z, Jelencic J, Bravar M, Marovic M. Influence of the network on the interaction parameter in system EPDM vulcanizate-solvent. Journal of Applied Polymer Science. 1997; 65: 991-999.

30 Brandrup J; Immergut EH; Grulke EA; Abe A; Bloch DR. Polymer handbook. 4ª edição. Nova lorque: John Wiley \& Sons, 1999.

31 Gestis Substance Database. n-Heptane.[acesso em 28 jan. 2014]. Disponível em: http://gestis-

en.itrust.de/nxt/gateway.dll/gestis_en/013820.xml?f=templates $\$ f n=d e f a u l t . h t m \$ 3.0$

32 César J, Paoli MA, Andrade JC. A determinação da densidade de sólidos e líquidos. Chemkeys; 2004.

33 Liang CY, Krimm S. Infrared spectra of high polymers. Part IX: Polyethylene Terephthalate. Journal of Molecular Spectroscopy, 1959; 3: 554-574.

34 Silverstein RM, Webster FX, Kiemle DJ. Spectrometric identification of organic compounds. $7^{\underline{a}}$ edição. Nova lorque: John Wiley \& Sons, 2005.

35 Forensic Science Services. Image gallery. [acesso em 10 fev. 2014] .Disponível em: http://www.forensicscienceservices.com/Image-Gallery.page

36 Choi S, Kim Y. Analysis of 5-ethylidene-2-norbornene in ethylene-propylene-diene terpolymer using pyrolysis-GC/MS. Polymer Testing. 2011; 30: 509-514.

37 Jia X, Li G, Sui G, Li P, Yu Y, Liu H, Yang X. Effects of pretreated polysulfonamide pulp on the ablation behavior of EPDM composites. Materials Chemistry and Physics. 2008; 112: 823-830

38 Paoli MA. Degradação e estabilização de polímeros. São Paulo: Artliber, 2009. 286 p..

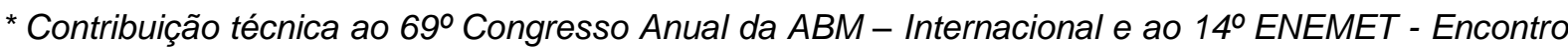
Nacional de Estudantes de Engenharia Metalúrgica, de Materiais e de Minas, 21 a 25 de julho de 2014, São Paulo, SP, Brasil.
} 